\title{
Emprendimiento Rural, una Aproximación desde el Empoderamiento Femenino
}

\author{
Rural entrepreneurship, an approach from female \\ empowerment
}

Ana María Castillo López; ${ }^{1}$ Daniel Ordóñez Abril; ${ }^{1}$ Lorena Erazo Bolaños; ${ }^{1}$ Jessica Cabrera Lozano; ${ }^{1}$

1 Fundación Universitaria Católica Lumen Gentium, Colombia

\section{Resumen}

En Colombia el emprendimiento rural y el empoderamiento femenino han tomado gran importancia, específicamente como una de las forma de independizarse y de obtener empleos las mujeres, dando muestras de sus capacidades físicas e intelectuales, que las convierten en agentes de progreso y de cambio de las condiciones de desigualdad. El objetivo principal del presente documento es efectuar una revisión de literatura sistemática sobre el empoderamiento femenino en el emprendimiento rural, siguiendo una metodología de búsqueda de referentes bibliográficos contextualizados en temas de empoderamiento femenino, emprendimiento, género, participación y liderazgo femenino. Se Encuentran diversos estudios centrados en analizar el emprendimiento femenino rural e indígena, la política pública con perspectiva de género, el empoderamiento femenino en las organizaciones y el desarrollo rural de las comunidades; las evidencias invitan a reflexionar sobre políticas de género y condiciones de derechos de las mujeres rurales, para disminuir la discriminación de la sociedad y estado.

Palabras clave: Emprendimiento-femenino; empoderamiento-femenino; participación femenina; liderazgo femenino; desarrollo rural

\begin{abstract}
In Colombia, rural entrepreneurship and female empowerment have taken on great importance, specifically as one of the ways for women to become independent and obtain jobs, showing their physical and intellectual capacities, which make them agents of progress and change for women. inequality conditions. The main objective of this document is to carry out a systematic literature review on female empowerment in rural entrepreneurship, following a search methodology for contextualized bibliographic references on issues of female empowerment, entrepreneurship, gender, participation and female leadership. There are various studies focused on analyzing rural and indigenous female entrepreneurship, public policy with a gender perspective, female empowerment in organizations and rural development of communities; the evidence invites reflection on gender policies and conditions of rural women's rights, to reduce discrimination in society and the state.
\end{abstract}

Keywords: Entrepreneurship-feminine; empowerment-feminine; feminine participation, feminine leadership; rural development

Cómo citar:

Castillo, A., Ordoñez, D., Erazo, L., \& Cabrera, J. (2020). Emprendimiento Rural, una Aproximación desde el Empoderamiento Femenino . Revista Empresarial, 14(1), 39-51. 


\section{INTRODUCCIÓN}

El empoderamiento femenino en el emprendimiento viene a tener sus primeras aproximaciones en la década de los sesenta con trabajos que abordaron el género como una construcción social que introdujo el imaginario social del rol que debe cumplir la mujer en la sociedad (Kargwell, 2012). Para la primera década del siglo XXI los estudios toman un giro enfocándose en las capacidades que tienen las mujeres en el desempeño de funciones, roles laborales y relaciones sociales (Pérez, Vásquez y Zapata, 2008). Lo que permitió que las políticas públicas a nivel global se orientaran a propender por la igualdad de género, como punto de desarrollo económico y laboral. Los estudios evidencian la necesidad de trabajar en la igualdad de condiciones para las mujeres en los diferentes ámbitos (Camberos, 2017). Por lo que se debe abordar a la mujer como sujeto de derechos que según Ordoñez y Aguilera (2019) está atada a en su cotidianidad a la exclusión en los escenarios públicos, reflejando constantes relaciones de poder que la subyugan y someten, pero, en las que no está completamente desvalida y puede resistirse. Por tanto, se habla de un poder inherente al ser humano que solo puede ser controlado a través de procesos continuos de análisis, autoanálisis y supervisiones.

Ahora bien, el empoderamiento económico de la mujer rural en los últimos años se ha estudiado desde el enfoque de procesos continuos ante el cambio y la desigualdad al acceso a sectores de distribución y producción, a los ingresos en el sector económico (por ejemplo, Manzanera y Lizarraga, 2013, y; Palacios, 2010). Lo que ha permitido analizar las características del emprendimiento rural femenino y el autoconsumo como sistemas de vida en estudios como los de Pallares y Tullas (2018). En este contexto, el género es abordado desde un proceso de modernización en el que la mujer se ha empoderado en lo social, y, adquiere una mayor participación en el espacio rural, confirmando el vínculo principal con el emprendimiento en economías locales y rurales (ver por ejemplo, Balcázar, 1998).

Es así como, la mujer debe ser estudiada como agente de cambio clave en lo rural. Ya que además de dedicarse al cuidado del hogar, es responsable de movilizar la actividad agrícola en diversos mercados. Lo que es evidenciado en estudios como los de Chong (2016) que identifican como la mujer ha sido participe de nuevas empresas relevantes en la segunda mitad del siglo XX, al tiempo que se ocupa de sus roles de madre y esposa. Sin embargo, se debe reconocer que con el surgimiento de la segunda ola feminista en los últimos años, se han abierto mayores oportunidades a las mujeres de incursionar en prácticas tradicionalmente masculinas. Empoderándola y liberándola para organizar actividades económicas en busca de expresar sus necesidades, capacidades y derechos (Ver por ejemplo; Rodríguez y Muñoz, 2015; Lombardo, 2008).

Por tanto, se puede validar estudios como los de Santander, Fernández y Yañez (2016), y; Queirol (2009) que muestran como el empoderamiento y el emprendimiento liderados o con participación de las mujeres son fundamentales para mejorar los niveles de pobreza y fortalecer un ambiente que promueve la actividad emprendedora y dinámica que aporta al crecimiento económico rural de un país. De ahí que, fue necesario revisar publicaciones relacionadas con modelos holísticos que relacionan variables psicosociales, al desempeño relacionado con el rol que tiene la mujer en el empoderamiento y el emprendimiento rural (ver algunos ejemplos Fernández, 2015; Didier y Luna, 2017; Lombardo, 2008; Figueroa y Forray, 2015; Martelo, Villanoba y Franco, 2019; Casique, 2004; Liñan, 2013; Montero y Camacho, 2018; Ortiz, 2017; Olaz y Ortiz, 2017, y; Forero, 2019).

A partir de lo anterior Emerge el siguiente cuestionamiento: ¿Cómo se puede caracterizar la literatura sistemáticamente sobre el empoderamiento femenino en el emprendimiento rural? De modo que este artículo presenta de manera panorámica, un repaso de la literatura sobre el empoderamiento femenino en el emprendimiento rural. Para lo que se utilizan como fuentes de datos, publicaciones de revistas indexadas de niveles Q1, Q2, Q3 y Q4, considerando los campos del empoderamiento femenino, 
la participación y el liderazgo femenino. Para lo que se adopta como método de repaso de literatura el planteado por Cronin, Ryan y Coughlan (2008).

El estudio se estructura a partir de cuatro secciones adicionales a la introducción. La primera, presenta la metodología empleada para la selección y búsqueda de literatura. La segunda, aborda conceptos básicos de la teoría del empoderamiento femenino. La siguiente sección presenta el análisis de los resultados y las discusiones. Por último, en las consideraciones finales se proponen, a la luz de los resultados encontrados, posibilidades de investigación en la temática a fin de trazar un panorama futuro para el estudio empoderamiento femenino y emprendimiento rural.

\section{METODOLOGÍA}

La metodología definida para lograr proporcionar una revisión sistémica de literatura que identifique, evalué y resuma los estudios pertinentes será la sugerida por Cronin et al. (2008). Que consiste en una serie de búsquedas en bases de datos, estableciendo prioridades en la selección del material y produciendo al final una síntesis del campo (Creswell, 2010). A continuación, se presenta el paso a paso de la investigación realizada.

\section{Selección del tópico de repaso}

El primer paso fue considerar dos conceptos, el empoderamiento femenino, basado en el concepto de poder planteado en el estudio de Ordoñez y Aguilera (2019), en el que el poder se emplea para delimitar y en el que la relación de poder significa actuar sobre otros, pero esos otros pueden oponerse. Por tanto, el concepto se relaciona con fundamentos teóricos como el comportamiento de los individuos en diferentes estamentos de poder y autoridad, delimitándose este poder con el autocontrol y la motivación. El segundo concepto fue el emprendimiento femenino visto desde la equidad de género y los roles que le son asignados en los ámbitos familiares, sociales, económicos y culturales. Ahora bien, para realizar un mejor análisis del emprendimiento se hace una exploración de la participación, el liderazgo de la mujer en los emprendimientos rurales. Esta selección del tópico se desarrolla a partir de lo dicho por Cronin et al. (2008) en cuanto "es mejor comenzar con un tema específico y, si es necesario, ampliar el alcance de la revisión a medida que avanza” (p. 39).

\section{Búsqueda de la literatura}

La búsqueda de la literatura se realizó en las bases de datos de mayor rigurosidad científica y académica como ScienceDirect, ISI - Web of Science, Springer, Emerald, Scielo y Google Scholar, ingresando descriptores de interés y ajustando los parámetros de búsqueda a empoderamiento femenino, emprendimiento rural, participación femenina, liderazgo femenino, female empowerment, female entrepreneurship, rural entrepreneurship, female participation y female leadership, para precisar los resultados. Priorizando los artículos publicados por journals de diferentes áreas con aceptación investigativa. Direccionándose todo el proceso sobre el empoderamiento femenino y emprendimiento rural, criterios que se tuvieron en cuenta para evitar problemas de dispersión de resultados (Cronin et al., 2008). Para llevar a cabo una recolección de datos eficaz se filtró la información principalmente por títulos y resúmenes de artículos publicados en los últimos treinta años.

\section{Recopilación de literatura, lectura y análisis}

La búsqueda de literatura inicial arrojo 106 artículos ubicados en las bases de datos científicas mencionadas anteriormente. Seleccionándose los artículos científicos que han sido debidamente validados (Podsakoff, MacKenzie, Bachrach, y Podsakoff, 2005), por 
tanto, se incluyeron expresiones de búsqueda asociadas con "empoderamiento femenino en el emprendimiento rural”. Después de esa primera exclusión, se mantuvieron los artículos científicos (80) publicados en revistas Q1, Q2, Q3 y Q4, que fueron preliminarmente recolectados.

En ese orden, se clasificaron los textos según el idioma; español, inglés y portugués y que estuvieran enfocados en los descriptores de búsqueda mencionados anteriormente. Siguiendo este procedimiento, se mantuvieron 52 artículos a los que se les hizo el respectivo análisis individualizado, teniendo en cuenta el enfoque de investigación; método; muestra cuantitativa y/o cualitativa; técnicas de recolección y análisis de datos; resultados y conclusiones. Para finalizar con el análisis de contenido y sistematización de los artículos en el software informático de análisis cualitativo Nvivo. Lo que permitió ver como el empoderamiento femenino en temas asociados con el emprendimiento es de interés para los diversos investigadores (ver Figura 1), y a los que se les ha realizado análisis en términos del tipo de estudio, tipo de análisis, conclusiones, alcance y similitudes entre los estudios.

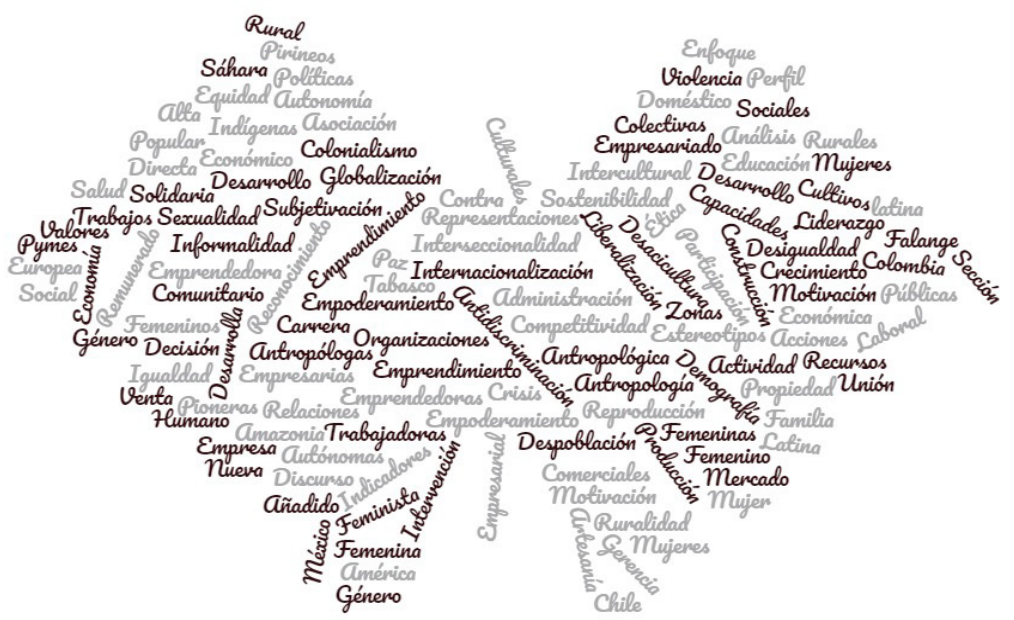

Figura 1. Representación de las 286 palabras (con más de cuatro letras) de los artículos analizados.

\section{RESULTADOS}

\section{Recopilación de literatura, lectura y análisis}

Desde el concepto de mujer y de acuerdo a lo planteado por Foucault refiriéndose al poder como un mecanismo de control sobre los individuos en una sociedad, se logra apreciar según Cano y Arroyave (2014) como en la historia las relaciones de las mujeres con los otros, se han caracterizado, por alguien que mandaba (hombre) y otras (ellas mismas) que obedecían; esto en relación con la suposición de que las mujeres estaban fuera de los ámbitos de poder, por lo que en sus relaciones cotidianas, otros (con más autoridad, dinero, oportunidades) dirigían sus vidas. Sin embargo, el empoderamiento de la mujer hoy en día se ha visto como un poder social que poco a poco ha ido rompiendo las brechas de las sociedades y de los entornos políticos alcanzado participación en su propia familia, el Estado, las organizaciones sociales, de lucha y por ende en las empresariales, teniendo en cuenta que conforme a la teoría de Foucault este poder que va ligado a las relaciones en una organización termina evidenciando el eje función-normas-estímulos (Ordoñez \& Aguilera, 2019). Este empoderamiento determina que las mujeres estén en procesos de toma de decisiones y términos de poder en un proceso individual y colectivo con el objetivo de lograr participar en igualdad de oportunidades en diferentes campos. 
Ahora bien, la participación femenina en el emprendimiento, refleja en la mujer su interés por explorar campos en desarrollo de su género y apropiarse de nuevos espacios y roles sociales (Queirol, 2009), percibiéndose el emprendimiento como un proceso más que como un evento, en el que las emprendedoras evalúan y persigue oportunidades de cambio (Palacios, 2010), Por lo que revisar el empoderamiento, la participación y el liderazgo de las mujeres en el emprendimiento, consiste en entenderla como agente de cambio vital para el desarrollo rural, razón por la cual, ha sido centro de diversos estudios (por ejemplo, Alberti, 1998; Pérez, Vásquez y Zapata, 2008; Casique, 2010; Bengochea, 2012; Sánchez, Jimenez, 2012; Manzanera, Lizárraga, 2013; Buendía, Carrasco, 2013; Cano, Arroyave, 2014; Saavedra, Camarena, 2015; Santamaria, 2015; Chong, 2016; Acosta, Zambrano, Suarez, 2017; Mora, Constanzo, 2017; Pérez, Tallón, 2017; Pallares, Vera, Tulla, 2018; Chamorro, 2020), centrados en la influencia de la mujer en el desarrollo de la comunidad, el territorio y las empresas.

En este orden de ideas se hace necesario definir el emprendimiento femenino, según Bartolomé (2018) como la evolución de la mujer en el ámbito laboral en una sociedad y su participación en la creación de empresas, en las que enfrentan retos financieros, familiares, culturales y sociales. En lo rural este emprendimiento trasciende según Guaneme (2019) al proyecto de vida desarrollado dentro de un territorio para contribuir al desarrollo del tejido social y económico de una región en base a la cultura y el aprovechamiento de recursos. Debe reconocerse que estos territorios son habitados por una gran cantidad de indígenas, por lo que se hace necesario entender también el emprendimiento indígena según Guerrero, Rivera, García \& Rico (2018) como una forma de organización económica en la que sus integrantes se unen para producir, intercambiar, comercializar, financiar, y consumir bienes y servicios que les permitan satisfacer sus necesidades y generar ingresos.

\section{Panorama de las Publicaciones}

La clasificación de las revistas científicas consultadas se relaciona en la Tabla 1. Se evidencia un compendio de publicaciones en revistas científicas con áreas de estudio enfocadas principalmente al empoderamiento y emprendimiento femenino, género y derecho de las mujeres rurales e indígena. Así, mismo se refleja una dispersión de revistas entre las que se destacan Revista Española de Ciencia Política con 5 artículos consultados, Cuadernos de Desarrollo Rural con 4 artículos y la Revista de estudios cooperativos con 3 artículos, al igual que la Revista de Estudios sociales, lo que equivale a una participación superior al $28 \%$ de las publicaciones consultadas. El resto de las revistas consultadas solo tuvieron dos y una publicación relevante frente a los temas consultados entre las que se destacan revistas como la Revista Austral de ciencias sociales; la Revista de economía mundial; Cuadernos de administración, y; Entramado, entre otras.

Ahora bien, el número de publicaciones sobre los temas de Empoderamiento de la mujer y emprendimiento femenino, demuestran que el desarrollo viene siendo constante en las principales revistas científicas internacionales y está muy relacionado con la antropología, sociología, psicología, administración y economía. En la Figura 2, se puede evidenciar un nivel de interés en las consultas sobre estos componentes acerca de la mujer, sobre todo en los últimos cinco años, donde se han realizado 29 publicaciones del total, mientras que los otros 23 artículos consultados fueron realizados en un espacio temporal de 17 años.

De igual forma, en la Tabla 1. Se muestra las consultas realizadas por categorías de las revistas, teniendo una mayor consulta las revistas ubicadas en el cuartil 3 con un $38 \%$ de las publicaciones, al igual que las ubicadas en el cuartil 4 con un $35 \%$, seguida por publicaciones ubicadas en el cuartil 2 con un $27 \%$ y cero registros en el cuartil 1 . 
Tabla 1. Cantidad de publicaciones por revistas indexadas y sus respectivas clasificaciones

\begin{tabular}{|c|c|c|c|}
\hline Clasificación & Revista & Cantidad & $\%$ \\
\hline \multirow[t]{9}{*}{ Q2 } & AIBR Revista de Antropología Iberoamericana & 1 & 1.9 \\
\hline & Diversitas Perspectivas en Psicología & 1 & 1.9 \\
\hline & RA \& DEM Revista de Administración y Dirección de empresas & 1 & 1.9 \\
\hline & Revesco - Revista de Estudios Cooperativos & 3 & 5.8 \\
\hline & Revista Colombiana de Antropología-Maguaré & 1 & 1.9 \\
\hline & Revista Colombiana de Psicología & 1 & 1.9 \\
\hline & Revista de Economía Mundial & 2 & 3.8 \\
\hline & Revista de Estudios Sociales & 3 & 5.8 \\
\hline & Revista Facultad de Ciencias Económicas & 1 & 1.9 \\
\hline \multirow[t]{13}{*}{ Q3 } & Cuadernos de administración & 1 & 1.9 \\
\hline & Cuadernos geográficos & 1 & 1.9 \\
\hline & Entramado & 2 & 3.8 \\
\hline & Espacios & 1 & 1.9 \\
\hline & Revista Aglala & 2 & 3.8 \\
\hline & Revista Austral de Ciencias Sociales & 1 & 1.9 \\
\hline & Revista de Ciencias Sociales (Ve) & 2 & 3.8 \\
\hline & Revista de Comunicación “VivatAcademia” & 1 & 1.9 \\
\hline & Revista Derecho Privado & 1 & 1.9 \\
\hline & Revista Española de Ciencia Política & 5 & 9.6 \\
\hline & Revista Mexicana de sociología & 1 & 1.9 \\
\hline & Revista Virtual Universidad Católica del Norte & 1 & 1.9 \\
\hline & Sholarship - Powered by the California Digital Library Univ. of California & 1 & 1.9 \\
\hline \multirow[t]{14}{*}{ Q4 } & Anfora & 1 & 1.9 \\
\hline & Arenal: Revista de historia de mujeres & 1 & 1.9 \\
\hline & Barataria Revista Castellano-Manchega de Ciencias Sociales & 1 & 1.9 \\
\hline & Clio \& Asociados & 1 & 1.9 \\
\hline & Cuadernos de Desarrollo Rural & 4 & 7.7 \\
\hline & Cuicuilco Revista de la escuela nacional de antropología e historia & 1 & 1.9 \\
\hline & Revista Criterio Libre & 2 & 3.8 \\
\hline & Revista de Antropología Social & 1 & 1.9 \\
\hline & Revista de Desarrollo Económico & 1 & 1.9 \\
\hline & Revista Española de Antropología Americana & 1 & 1.9 \\
\hline & Revista Facultad de Ciencias Económicas & 1 & 1.9 \\
\hline & Revista Galega de Economía & 1 & 1.9 \\
\hline & Revista Lusófona de Educação & 1 & 1.9 \\
\hline & Sotavento MBA & 1 & 1.9 \\
\hline
\end{tabular}

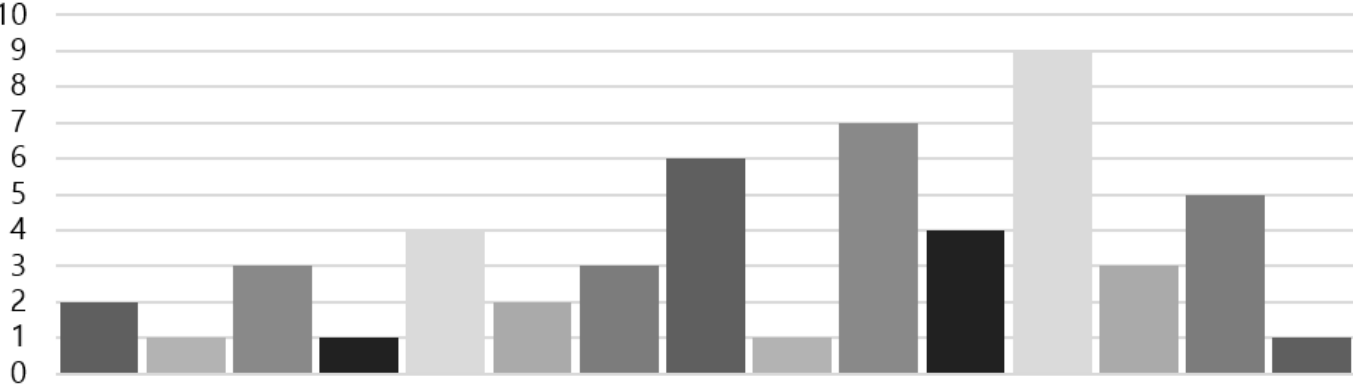

199820042008200920102011201220132014201520162017201820192020

Figura 2. Número de publicaciones de artículos por año con temas enfocados al empoderamiento y emprendimiento femenino 


\section{Panorama de las Publicaciones}

Se realizó un análisis exploratorio del contenido de los estudios analizados. La Figura 1 muestra la representación de las 286 palabras con más de cuatro letras que se mencionaron con mayor frecuencia en estos artículos. Destacándose las palabras como empoderamiento femenino, emprendimiento femenino, participación femenina y liderazgo femenino, de acuerdo con el área de estudio, pero que son íntimamente relacionadas con otras palabras de nivel secundario tales como informalidades, crecimiento, empoderamiento, equidad, crecimiento laboral, autonomía, independencia, lo que se demostrará más adelante en el artículo. Por otra parte, después de analizar las fechas de los artículos, que varían desde 1998 al 2019, se encuentra que la mayoría aparece en los últimos cinco años, lo que evidencia la creciente importancia de este tema.

La Figura 2 presenta los resultados de los artículos observados en términos de la similitud de las palabras utilizadas en función del coeficiente de correlación de Pearson. Como se observó, el estudio permite la identificación de varios grupos de artículos que resultan más relacionados entre sí cuando la figura se lee de abajo hacia arriba. De la misma manera, se entiende cómo hay claramente dos grupos principales y mutuamente distintos. El primer grupo de artículos (identificado en la parte superior de la Figura 2), sobre todo abarca artículos que estudian el empoderamiento femenino. Mientras que el clúster que contiene el resto de los artículos relaciona el emprendimiento femenino rural con los temas de participación y el liderazgo femenino.

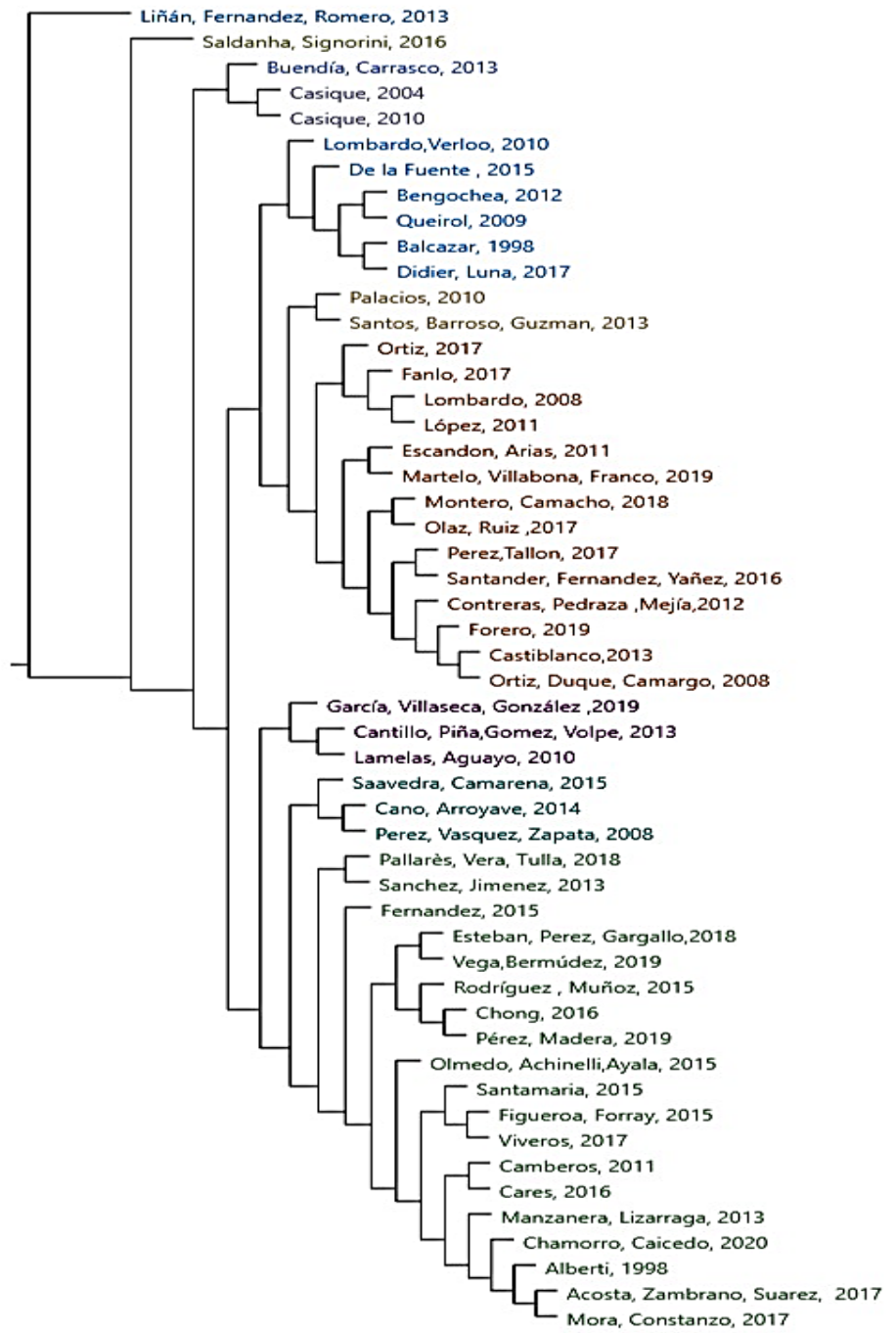

Figura 3. Agrupación de artículos por similitud de palabra 
Tabla 1. Cantidad de publicaciones por revistas indexadas y sus respectivas clasificaciones

\begin{tabular}{|c|c|c|}
\hline $\begin{array}{l}\text { Tema de } \\
\text { análisis }\end{array}$ & Principales conclusiones & Estudios \\
\hline $\begin{array}{l}\text { Empode- } \\
\text { ramiento } \\
\text { Femenino }\end{array}$ & $\begin{array}{l}\text { Se evidencia desde el enfoque del trabajo la informali- } \\
\text { dad y la precarización del trabajo: un trabajo altamente } \\
\text { dependiente, desregulado, intensivo, que entraña } \\
\text { riesgos de bajos ingresos e incierto, que aprovecha } \\
\text { una serie de ambigüedades subjetivas apoyándose en } \\
\text { los discursos empresariales del empoderamiento y el } \\
\text { emprendimiento femenino. }\end{array}$ & $\begin{array}{l}\text { Vega, Bermúdez (2019). Manzanera, Li- } \\
\text { zárraga (2013). Pérez, Vázquez, Zapata, } \\
\text { (2008), Camberos, (2017), Saldanha, Sig- } \\
\text { norini, (2016), Casique, (2010), Balcázar, } \\
\text { (1998), Saavedra, Camarena, (2015), Cano, } \\
\text { Arroyave (2014), Olmedo, Achinelli (2015). }\end{array}$ \\
\hline $\begin{array}{l}\text { Género y Em- } \\
\text { prendimiento }\end{array}$ & $\begin{array}{l}\text { - El problema que se observa en la economía mundial } \\
\text { es que el tipo de emprendimiento que se promueve es } \\
\text { el que persigue fundamentalmente la maximización de } \\
\text { beneficios, en coherencia con el paradigma competitivo. } \\
\text { - En este sentido, el cooperativismo ofrece oportuni- } \\
\text { dades para el desarrollo personal y profesional de las } \\
\text { mujeres rurales. } \\
\text { - El emprendimiento como actividad exigente especial- } \\
\text { mente para las mujeres debido a las problemáticas que } \\
\text { genera los estereotipos de género y los diferentes roles } \\
\text { que ejerce la mujer en la sociedad. } \\
\text { - La brecha de género se ha reducido en los aspectos } \\
\text { relacionados con el emprendimiento femenino, pero } \\
\text { es necesario vincular y producir redes que aporten } \\
\text { valor a las mujeres emprendedoras para consolidar sus } \\
\text { negocios. }\end{array}$ & $\begin{array}{l}\text { Alberti (1998), Escandón, Arias (2011), Li- } \\
\text { ñan, Fernández, Romero (2013), Santos, Ba- } \\
\text { rroso, (2013), Pérez, Tallón (2017), Casique, } \\
\text { (2004), Pérez, Madera, (2019), Martelo, Vi- } \\
\text { llabona, Franco (2019), Montero, Camacho } \\
\text { (2018), García, Villaseca, González (2019), } \\
\text { Cantillo, Piña, Gómez, Volpe (2013), Pala- } \\
\text { cios, (2010), Ortiz (2017), Olaz, Ortiz (2017), } \\
\text { Castiblanco (2013), Ortiz, Duque, Camargo } \\
\text { (2008), Buendía, Carrasco, (2013), Forero, } \\
\text { (2019), Viveros (2017), Acosta, Zambrano, } \\
\text { Suarez (2017), Mora, Constanzo (2017). }\end{array}$ \\
\hline $\begin{array}{c}\text { Participación } \\
\text { Femenina }\end{array}$ & $\begin{array}{l}\text { - La participación económica femenina en el espacio } \\
\text { rural tiene diversas implicaciones, la economía local se } \\
\text { mueve hacia sectores distintos a los tradicionales. } \\
\text { - No solamente las mujeres ayudan a los trabajos “no } \\
\text { remunerados en el campo", sino que ahora se incorporan } \\
\text { a otro sector donde son asalariadas en su minoría y } \\
\text { trabajadoras por cuenta propia en su mayoría, lo que les } \\
\text { permite tener una independencia hacia su autonomía } \\
\text { económica. } \\
\text { - Los artículos evidencian cuatro modos en que la teoría } \\
\text { feminista ha abordado, de forma explícita o implícita esta } \\
\text { temática, desde la perspectiva del poder: El poder como } \\
\text { recurso y como dominación, el poder como cuidado y el } \\
\text { poder como libertad. }\end{array}$ & $\begin{array}{l}\text { Chong, (2016), De la Fuente, (2015), Di- } \\
\text { dier, Luna (2017), Lombardo, Verloo } \\
\text { (2010), Figueroa, Forray (2015), Contre- } \\
\text { ras, Pedraza, Mejía (2012), Fanlo (2017), } \\
\text { López (2011), Lombardo (2008), Palla- } \\
\text { res, Vera, Tulla (2018), Lamelas, Aguayo } \\
\text { (2010), Cares, (2016), Bengochea, (2012), } \\
\text { Rodríguez, Muñoz, (2015), Esteba, Pé- } \\
\text { rez, Gargallo (2018), Chamorro (2020). }\end{array}$ \\
\hline $\begin{array}{l}\text { Liderazgo } \\
\text { Femenino }\end{array}$ & $\begin{array}{l}\text { - Es necesario realizar un análisis a partir de una nueva } \\
\text { división entre las mujeres, las que quieren diseñar es- } \\
\text { trategias de emancipación adecuadas que incrementen } \\
\text { el poder colectivo y no refuercen los ejes de separación } \\
\text { entre unas, y otras, para así diseñar estrategias de eman- } \\
\text { cipación adecuadas que incrementen una propuesta } \\
\text { política feministas. } \\
\text { - La variedad de experiencias vitales que nos atraviesan y } \\
\text { entre las cuales los feminismos han de hacer un esfuerzo } \\
\text { por identificar los nexos de unión entre mujeres si quie- } \\
\text { ren diseñar estrategias de emancipación adecuadas que } \\
\text { incrementen el poder colectivo y no refuercen los ejes de } \\
\text { separación entre unas y otras. }\end{array}$ & $\begin{array}{l}\text { Santander, Fernández, Yáñez (2016), } \\
\text { Fernández, (2015), Queirol, (2009), Sán- } \\
\text { chez, Jiménez (2012), Santamaría, (2015). }\end{array}$ \\
\hline
\end{tabular}

El análisis de las tablas confirman la relación entre los artículos basados en temas vinculados al emprendimiento femenino y el empoderamiento femenino con un enfoque hacia la ruralidad, reflejándose esto en la distancia entre los textos, por ejemplo, artículos como el Saldanha, Signorini, (2016) expresan que el empoderamiento se debe comprender como un proceso que a veces es individual y otras veces colectivo, los artículos de Buendía y Carrasco (2013), Santamaria (2015), Santos, Barroso \& Guzman (2013), vinculan a las mujeres en diversos roles y ámbitos de desempeño: académico, social, económico y cultural, así como los cambios que ello ha generado en las relaciones, rupturas y deterioro del poder masculino en su cotidianidad. En esta misma línea se encuentran artículos como el de Acosta, Zambrano \& Suárez, (2017) que basan su investigación en la mujer como agente de cambio en la economía rural y en las condiciones de desigualdad imperantes en sus comunidades. Esta investigación también consideró los 
temas estudiados y sus respectivas conclusiones claves (Tabla 2). Mostrando el interés de realizar estudios sobre la mujer en temas de empoderamiento y el emprendimiento femenino en las últimas décadas.

Referente a los estudios sobre el empoderamiento femenino, género y emprendimiento, participación y liderazgo femenino, se destacan las actitudes, normas subjetivas, saberes, conocimientos y percepciones sobre el control del comportamiento como variables adecuadas para explicar el comportamiento emprendedor de las mujeres residentes en zonas rurales, hallándose líneas de investigación como: a) estudios sobre el empoderamiento femenino, b) estudios sobre el emprendimiento femenino, c) estudios sobre la participación y el liderazgo femenino, y, d) estudios sobre la desigualdad y derechos de la mujer. Que finalmente concluyen que las intenciones y comportamientos pueden predecirse a partir de la actitud, el control percibido y las normas subjetivas, enmarcados en una perspectiva decolonial para el análisis de aspectos como las relaciones de poder, el saber y el ser.

En ese orden de ideas el empoderamiento femenino en los estudios analizados parte de las representaciones sociales de género, desde su cosmovisión y ofreciendo una perspectiva diferente de concebir a la mujer dentro del mercado laboral (Camberos Sánchez, 2017), así el trabajo de la mujer fuera del hogar y su empoderamiento se ven como mecanismos para la superación de las inequidades de género y el desarrollo del potencial individual de las mujeres (Casique, 2004), facilitando la actividad emprendedora femenina y el desarrollo de los países, y de manera indirecta, el desarrollo rural (Buendía y Carrasco, 2013, y; Olmedo y Achinelli, 2015). De la misma manera, se encuentra en los estudios que las mujeres se asocian para compartir y satisfacer sus intereses estratégicos de género (Manzanera \& Lizarraga, 2013), por tanto, los procesos de empoderamiento se constituyen en modos de subjetivación en tanto las mujeres se apropian de estos a través de sus propias experiencias, deseos y limitaciones (Cano \& Arroyave, 2014). Subrayando la importancia de promover políticas y legislaciones que favorezcan el acceso equitativo de hombres y mujeres a los recursos, y disponer de indicadores que den seguimiento a esta situación (Casique, 2010).

Por otro lado, en varios de los estudios se identificaron variables que hacen parte del constructo de competitividad de las empresas creadas por mujeres (Escandón \& Arias, 2011), en las que la organización ha sido uno de los principales impulsores para transformar la situación de las mujeres, a partir de su autovaloración, su participación en el rumbo de la empresa y en el control de los recursos materiales y simbólicos (Alberti, 1998), lo que finalmente lleva a pensar que la incorporación de las mujeres al ámbito productivo coinciden con el papel que juegan los programas de emprendimiento asociativo en las zonas rurales impulsados por el sector público (Mora \& Constanzo, 2017).

Es decir, los estudios realizados muestran que el fomento de cooperativas femeninas se configuran como un importante y afianzado actor de la vida local rural (Sánchez \& Jiménez, 2012), y favorece la igualdad entre hombres y mujeres, puesto que la participación en iniciativas que ofrecen independencia económica constituye un elemento crucial para avanzar hacia la igualdad de género (Pérez \& Tallón, 2017), lo que conlleva a afirmar que la participación laboral de la mujer, en especial, del sector rural, constituye un importante acontecimiento transformador del actual mercado de trabajo (Rodríguez \& Muñoz, 2015). Por tanto, hay que tener un enfoque integrador que ponga en perspectiva de género lo económico y lo político en conversación con lo cultural. Desde allí, la praxis reflexiva del profesional debe incidir en la construcción de estas asociaciones para posicionar a las mujeres, en especial en contextos rurales afectados por las violencias (Chamorro, 2020).

Esta revisión respecto al liderazgo femenino deja más interrogantes que certezas en cuanto al futuro. Aunque las mujeres han alcanzado un importante posicionamiento a nivel laboral, en comparación con décadas anteriores, aún existen importantes brechas que les limita la posibilidad de alcanzar cargos de alta dirección (Contreras, Pedraza \& Mejia, 2012), lo que marca una nueva división entre las mujeres que quieren diseñar estrategias de emancipación adecuadas que incrementen el poder colectivo y no reforzar los ejes de separación entre unas y otras (Fernández, 2015) 
Finalmente, se analizó la tipología de los estudios con respecto al tipo de análisis realizado (Tabla 3). En la revisión teórica se evidencian 17 estudios, De los estudios empíricos que corresponden a los cuantitativos 8 son de análisis estadísticos descriptivos, 2 son de análisis de ecuaciones estructurales, y 1 de análisis de modelo de revisión logística. De los estudios empíricos cualitativos, 18 son de análisis de contenido, 5 de análisis empírico descriptivo y 1 de análisis de estudio de casos. El análisis de la Tabla 3 confirma que las metodologías cualitativas son las de mayor aplicación en este campo. Sin embargo, se evidencia escases de estudios mixtos que permitan realizar investigaciones más integrales y multicomplementarias, lo que debe ser el objetivo de futuras investigaciones.

\section{DISCUSIONES}

A partir de los resultados presentados por los diferentes estudios, y analizando más allá del componente estructural de esta investigación, surgieron diversas ideas e inquietudes sobre los documentos consultados. Los aportes concluyen en la necesidad de fortalecer temas de investigación enfocados hacia el emprendimiento rural de las mujeres, dado que tal como lo expresa Palacios, (2010) por medio de un emprendimiento social como modelo innovador se puede enfrentar la exclusión rural, pues los pequeños productores, pueden adoptar prácticas que se les han negado pero que a través de sus modelos de emprendimiento logran un rol importante en la expansión del mismo, mostrando que esta clase de emprendimientos pueden ser alternativas para superar la exclusión en comunidades rurales e indígenas y en especial de las mujeres.

En otras palabras, las expectativas que se tienen respecto a los beneficios de la incorporación laboral de las mujeres y del proceso de auto empoderamiento de las mismas son

Tabla 3. Tipo de Estudio y Tipo de Análisis

\begin{tabular}{|c|c|c|}
\hline $\begin{array}{l}\text { Tipo de } \\
\text { estudio }\end{array}$ & Tipo de análisis & Estudios \\
\hline $\begin{array}{l}\text { Revisión } \\
\text { teórica }\end{array}$ & & $\begin{array}{l}\text { Lombardo, Verloo (2010), De la Fuente (2015), López (2011), Lom- } \\
\text { bardo (2008), Forero (2019), Castiblanco (2013), Fernández (2015), } \\
\text { Queirol (2009), Cares (2016), Saavedra, Camarena (2015), Fanlo } \\
\text { (2017), Chamorro (2020), Casique (2004), Cano, Arroyave (2014), } \\
\text { Olmedo, Achinelli (2015), Santamaria (2015), Balcazar (1998). }\end{array}$ \\
\hline \multirow[t]{6}{*}{$\begin{array}{l}\text { Estudios empí- } \\
\text { ricos }\end{array}$} & $\begin{array}{l}\text { Cuantitativos } \\
\text { Análisis estadísticos des- } \\
\text { criptivos }\end{array}$ & $\begin{array}{l}\text { Pallarès, Vera, Tulla (2018), Buendía, Carrasco (2013), Cantillo, Piña, Gó- } \\
\text { mez, Volpe (2013), Lamelas, Aguayo (2010). Figueroa, Forray (2014), Di- } \\
\text { dier, Luna (2017), Pérez, Madera (2019), Esteban, Pérez, Gargallo (2018). }\end{array}$ \\
\hline & Análisis EQS & Liñan, Fernández, Romero (2013), Montero, Camacho (2018). \\
\hline & $\begin{array}{l}\text { Análisis de modelo de } \\
\text { revisión logística }\end{array}$ & Rodríguez, Muñoz (2015). \\
\hline & $\begin{array}{l}\text { Cualitativos } \\
\text { Análisis de contenido }\end{array}$ & $\begin{array}{l}\text { Ortiz (2017), Olaz, Ortiz (2017), Contreras, Pedraza, Mejía, (2012). } \\
\text { Pérez, Tallón (2017), Acosta, Zambrano, Suarez (2017), Vega, Ber- } \\
\text { múdez (2019), Santander, Fernández, Yañez (2016), Manzanera, Li- } \\
\text { zárraga (2013), Pérez, Vázquez, Zapata (2008), Bengochea (2012), } \\
\text { Saldanha, Signorini (2016), Casique (2010), Viveros (2017), Balcázar } \\
\text { (1998), Sánchez, Jiménez (2012), Martelo, Villabona, Franco (2019), } \\
\text { Mora, Constanzo (2017). Escandón, Arias (2011). Chong (2016). }\end{array}$ \\
\hline & Análisis empírico descritpivo & $\begin{array}{l}\text { García, Villaseca, González (2019). Santos, Barroso (2013), Or- } \\
\text { tiz, Duque, Camargo (2008). Camberos (2017), Alberti (1998). }\end{array}$ \\
\hline & Estudio de caso & Palacios (2010). \\
\hline
\end{tabular}


amplias. Sin embargo, se puede considerar hacer estudios desde, perspectivas feministas sobre el tema dado que permitirían profundizar en aspectos relacionados con los avances en las relaciones de poder de las mujeres en la sociedad. En el desarrollo de este artículo se evidencia, el rol de una mujer que proporciona progreso y prosperidad a su entorno social y económico, al participar de comunidades, empresas y organizaciones a pesar de las exclusiones a las que aún está sometida.

Es decir, la revisión permite apreciar que los estudios están centrados en comprender los aspectos relacionados con el empoderamiento y emprendimiento femenino, en cuyo caso, la mujer indígena en las zonas rurales se ha convertido en un agente de cambio importante, pues, ella tiene gran participación y poder en los ámbitos sociales, económicos y del hogar. Por tanto, se considera que el emprendimiento rural debe tener un mayor alcance de investigación y debe ser enfocado a las comunidades indígenas, de tal manera que las futuras líneas de investigación sean, por un lado, en emprendimiento rural indígena, y, por otro, en temas desarrollados con los avances del poder de la mujer en la sociedad.

Dichas líneas de investigación implican entender que el espíritu empresarial debe ser formado desde jóvenes a través de sistemas educativos que logren relacionar el empoderamiento femenino con el emprendimiento en cuanto a la toma de decisiones (Riaga, Mayorga \& Orozco, 2008). Según esto, y teniendo en cuenta las observaciones realizadas en el desarrollo del documento sobre el empoderamiento femenino, el emprendimiento rural, la participación y el liderazgo femenino, se destacan los comportamientos en el desempeño que tienen las mujeres en diferentes cargos o empleos, el modelo y percepción del comportamiento sobre el control que han venido realizando sobre cualidades, experiencias, estrategias, normas y políticas sobre los roles que debe desempeñar, y la participación en el nivel socio económico de un país, por todo esto se propone la necesidad de trabajar las líneas de investigación con enfoques mixtos y desde diferentes perspectivas disciplinares incluyendo perspectivas críticas como el feminismo. Que validen y analicen intenciones y comportamientos en ambientes que promuevan la actividad y el crecimiento socio-económico de un país.

\section{CONCLUSIONES}

El desarrollo de este artículo de investigación permitió crear un amplio panorama literario sobre las conceptos, características y aspectos importantes relacionados con las mujeres en cuanto a la manera en que se han ido empoderando y a su vez generando emprendimiento en un espacio en el que persiste desigualdad de género y como de esta manera su papel ha tomado importancia en el desarrollo económico y social de las organizaciones y comunidades, lo que permitió visibilizar un panorama sobre estos conceptos y así propiciar aportes para el emprendimiento rural de las mujeres indígenas y como materia de consulta para organizaciones de mujeres con fines sociales, económicos y productivos. No obstante, en la literatura analizada se encontraron rasgos que indica que aún existen muchos paradigmas y brechas por romper y trabajar, ya que en esta revisión literaria son poco los artículos y/o investigaciones que tienen acercamiento investigativo con las mujeres rurales y las indígenas desde una perspectiva feminista, también se pudo evidenciar que falta material investigativo relacionado con la necesidad de los gobiernos en establecer políticas públicas que favorezcan el Desarrollo de la mujeres en especial las rurales, como también las garantías de las mismas para sus proyectos de desarrollo rural.

La literatura analizada muestra que para la mayoría de las mujeres su principal motivación para emprender es mejorar su condición de vida, ya que al faltar empleo en sus municipios, su edad y su falta de formación académica les impide conseguir un trabajo que se adapte a sus condiciones personales y familiares, haciéndose necesario profundizar en el campo femenino rural. De ahí que se pueda concluir que los estudios acerca del emprendimiento femenino coinciden en que, en su mayoría, las investigaciones se han dedicado a reconocer las diferencias entre los emprendimientos de mujeres pero pocos de ellos se han enfatizado en entender cuáles son necesidades reales de las mujeres como ser psicológico y social capaz de aportar y el contexto que implica cada emprendimiento. Se espera que a partir del este artículo las mujeres y en especial las rurales e indígenas puedan ampliar sus conocimientos respecto a sus derechos y su ser de género y con ellos el favorecimiento del desarrollo rural que se desea alcanzar. 


\section{REFERENCIAS}

Acosta, B., Zambrano, S., Suárez, M. (2017). Emprendimiento femenino y ruralidad en Boyacá, Colombia. Criterio Libre 215-236. https://doi.org/10.18041/1900-0642/criteriolibre.2017v15n26.1048

Alberti, P. (1998). La organización de mujeres indígenas como instrumento de cambio en el desarrollo rural con perspectiva de género. Revista Española de Antropología Americana, 28, 189-213. https://revistas.ucm.es/index.php/ REAA/article/view/REAA9898110189A

Balcázar, C. (1998). Poder y empoderamiento de las mujeres. Revista de Estudios Sociales, (1), 131-132. https://journals.openedition.org/revestudsoc/31593

Bartolomé, L. (2018). El emprendimiento femenino creció un 13\% a nivel mundial en 2017. Obtenido de https://www. eleconomista.es/gestion-empresarial/noticias/9344721/08/18/Elemprendimiento-femenino-crecio-un-13-a-nivel-mundial-en-2017.html

Bengochea, E. (2012). Las mujeres saharauis a través de la Sección Femenina, un sujeto colonizado. Arenal: Revista de historia de mujeres, 19(1), 143-159. https://revistaseug.ugr.es/index.php/arenal/article/view/1411/0

Buendía I., \& Carrasco, I. (2013). Mujer, actividad emprendedora y desarrollo rural en América Latina y el Caribe. Cuadernos de desarrollo rural, 10(72), 21-45. https://revistas.javeriana.edu.co/index.php/desarrolloRural/article/ view/7006

Camberos, M. (2011). Empoderamiento femenino y políticas públicas, una perspectiva desde las representaciones sociales de género. Entramado, 7(2), 40-53. https://revistas.unilibre.edu.co/index.php/entramado/article/view/3400

Cano, T., \& Arroyave, O. (2014). Procesos de empoderamiento de mujeres: subjetivación y transformaciones en las relaciones de poder. Revista Virtual Universidad Católica del Norte, 2(42), 94-110. https://revistavirtual.ucn.edu. co/index.php/RevistaUCN/article/view/497/1047

Cantillo, E., Pina, O., Gómez, A., \& Volpe, I. (2013). El Efecto De La Formación En Emprendimiento Sobre La Construcción De Una Mentalidad Emprendedora En Estudiantes De Ingeniería Industrial En Una Universidad De La Ciudad De Barranquilla. Sotavento MBA, (21). https://revistas.uexternado.edu.co/index.php/sotavento/ article/view/3436

Cares, C. (2016). Alcances de la perspectiva de género en la política cultural chilena. Revista Austral de Ciencias Sociales, (30), 52-76. http://revistas.uach.cl/index.php/racs/article/view/864

Casique, I. (2004). Trabajo femenino, empoderamiento y bienestar de la familia. Sholarship - Powered by the California Digital Library-University of California. https://escholarship.org/uc/item/6zr8t8sw

Casique, I. (2010). Factores de empoderamiento y protección de las mujeres contra la violencia. Revista mexicana de sociología, 72(1), 37-71. http://www.scielo.org.mx/pdf/rms/v72n1/v72n1a2.pdf

Castiblanco, S (2013). La construcción de la categoría de emprendimiento femenino. Revista Facultad de Ciencias Económicas, 21(2), 53-66. https://www.redalyc.org/pdf/909/90930501005.pdf

Chamorro-Caicedo, L. S. (2020). Acercamientos a asociaciones de mujeres campesinas en Colombia y proyecto ético-político del Trabajo social. ÁNFORA, 27(48), 189-214. https://publicaciones.autonoma.edu.co/index.php/ anfora/article/view/674

Chong, E. G. (2016). El emprendedurismo femenino rural. Entramado, 12(1), 30-36. http://www.scielo.org.co/pdf/ entra/v12n1/v12n1a03.pdf

Contreras, F., Pedraza, J., \& Mejía, X. (2012). La mujer y el liderazgo empresarial. Diversitas: Perspectivas en Psicología, 8(1), 183-194. https://www.redalyc.org/pdf/679/67923973012.pdf

Creswell, J. (2010). Mapping the developing landscape of mixed methods research. SAGE handbook of mixed methods in social \& behavioral research, 2, 45-68.

Cronin, R. \& Coughlan, M. (2008). Undertaking a literature review: a step-by-step approach. British journal of nursing, $17(1), 38-43$.

De la Fuente, M. (2015). Ideas de poder en la teoría feminista. Revista Española de Ciencia Política, (39), 173-193. https://recyt.fecyt.es/index.php/recp/article/view/38504

Didier, N., \& Luna, J. F. (2017). ¿Dónde estamos? La cultura laboral chilena desde Hofstede. Revista Colombiana de Psicología, 26(2), 295-311. http://www.scielo.org.co/pdf/rcps/v26n2/0121-5469-rcps-26-02-00295.pdf

Escandón, D., \& Arias A. (2011). Factores que componen la competitividad de las empresas creadas por mujeres y las relaciones entre ellos. Cuadernos de administración, 24(42). https://www.redalyc.org/pdf/205/20520042008.pdf

Esteban, M., Pérez, F., \& Gargallo, A. (2018). Áreas rurales y cooperativas: iniciativas de mujeres para el desarrollo. Revesco: revista de estudios cooperativos, (127), 116-138. https://revistas.ucm.es/index.php/REVE/article/ view/58397

Fanlo, I., (2017). Derechos reproductivos y libertad de las mujeres. Observaciones sobre el debate feminista. Rev. Derecho Privado, 32, 29. https://revistas.uexternado.edu.co/index.php/derpri/article/view/5020

Fernández, A., (2015). Silvia Federici: Revolución en punto cero. Trabajo doméstico, reproducción y luchas feministas. Revista Española de Ciencia Política. (39), 301-305. https://recyt.fecyt.es/index.php/recp/article/view/39865

Figueroa, C., \& Forray, R. (2015). Movilidad femenina: Los reveses de la utopía socioespacial en las poblaciones de Santiago de Chile. Revista de Estudios Sociales, (54), 52-67. http://www.scielo.org.co/scielo.php?pi$\mathrm{d}=$ S0123-885X2015000400005\&script=sci abstract\&tlng=es

Forero, L., (2019). Así somos y así salimos adelante, un acercamiento al perfil de la mujer emprendedora. Revista espacios, 40(27). http://www.revistaespacios.com/a19v40n27/19402728.html

García, J., Villaseca, D., \& González, S. (2019). Emprendimiento femenino y financiación social: un estudio comparado. Revesco. Revista de Estudios Cooperativos, (132), 97-121. https://revistas.ucm.es/index.php/REVE/article/ view/65974

Guaneme Rincón, L. A. (2019). Emprendimiento rural enfocado al desarrollo económico en la región sabana centro (Doctoral dissertation)

Guerrero, Rivera, García, \& Rico, (2018). Economía social: Sumak Kawsay y empoderamiento de la mujer. Economía, 39(32). https://www.revistaespacios.com/a18v39n32/a18v39n32p34.pdf 
Kargwell, S. A. (2012). Women entrepreneurs breaking through: Push and pull within UAE cultural context. International journal of business and social science, $3(17)$.

Lamelas, N. \& Aguayo, E. (2010) Desigualdad de género en Latinoamérica desde las perspectivas de los indicadores de desarrollo humano. Revista Galega de Economía, 19 (2) 1-15. http://www.usc.es/econo/RGE/Vol19_2/castelan/art4c.pdf

Liñán, F., Fernández, J., \& Romero, I. (2013). Emprendimiento por necesidad y por oportunidad: El efecto mediador de la cultura. Revista de Economía Mundial, (33), 21-48. https://www.sem-wes.org/es/node/490

Lombardo, E. (2008). Desigualdad de género en la política: un análisis de los marcos interpretativos en España y en la Unión Europea. Revista Española de Ciencia Política, 18, 95-120. https://webs.ucm.es/info/target/Art\%20Chs\%20 ES/ArtPolESUE_RECP_08ES.pdf

Lombardo, E., \& Verloo, M. (2010). La 'interseccionalidad' del género con otras desigualdades en la política de la Unión Europea. Revista Española de Ciencia Política (23), 11-30. https://recyt.fecyt.es/index.php/recp/article/ view/37496

López, S., (2011). ¿Cuáles son los marcos interpretativos de la violencia de género en España?: Un análisis constructivista. Revista Española de Ciencia Política, (25), 11-30. https://recyt.fecyt.es/index.php/recp/article/view/37513

Manzanera, R., \& Lizárraga, C. (2013). Acciones colectivas femeninas y empoderamiento económico en la comunidad de Soni (Tanzania). AIBR. Revista de Antropología Iberoamericana, 8(2), 233-259. https://www.redalyc.org/ pdf/623/62329867005.pdf

Martelo, Z.., Villabona, N., \& Franco, D. (2019). Estrategias que apoyan el emprendimiento y el crecimiento sostenible. Aglala, 10(1), 67-86. https://dialnet.unirioja.es/servlet/articulo?codigo=7075900

Montero, B., \& Camacho, J. (2018). Caracterización del emprendimiento femenino en España: Una visión de conjunto. Revesco. Revista de Estudios Cooperativos, (129), 39-65. https://revistas.ucm.es/index.php/REVE/article/ view/ 61936

Mora, G. \& Constanzo, J. (2017). 'Emprender sin descuidar la casa': Posiciones y dinámicas organizativas en una asociación productiva de mujeres rurales. Cuadernos de Desarrollo Rural, 14(80), 90-107. https://revistas.javeriana. edu.co/index.php/desarrolloRural/article/view/20822

Olaz, A., \& Ortiz, P. (2017). Aproximación a la caracterización del emprendimiento femenino: una investigación cualitativa en clave competencial. Barataria: Revista castellano-manchega de ciencias sociales, (22), 51-66. https:// www.revistabarataria.es/web/index.php/rb/article/view/335

Olmedo, S., Achinelli, M., \& Ayala, D. (2015). Empoderamiento económico de las mujeres artesanas paraguayas en los departamentos de central, Guairá y Cordillera. Un análisis a través del estado del arte. Revista de Desarrollo Económico, 2(4), 254-265. https:/www.researchgate.net/publication/311678385_Empoderamiento_Economico_ de_las_Mujeres_Artesanas_Paraguayas_en_los_Departamentos_de_Central_Guaira_y_Cordillera

Ordoñez, D., \& Aguilera, C. (2019). El Poder En la Organización Desde La Perspectiva Psicoanalítica Revista Universitaria Ruta, 21 (2), 13-37. https://revistas.userena.cl/index.php/ruta/article/view/1251

Ortiz, P. (2017). El discurso sobre el emprendimiento de la mujer desde una perspectiva de género. revista de comunicación 'vivat academia', (140). https://www.vivatacademia.net/index.php/vivat/article/view/1038

Ortiz, C., Duque, Y. \& Camargo, D. (2008). Una revisión a la investigación en emprendimeinto femenino. Revista Facultad de Ciencias Económicas: investigación y reflexión, 16(1), 85-104. http://www.scielo.org.co/pdf/rfce/v16n1/ v16n1a07.pdf

Palacios, G. (2010). Emprendimiento social: integrando a los excluidos en el ámbito rural. Revista de Ciencias Sociales (Ve), 16(4), 579-590. https://www.redalyc.org/pdf/280/28016613002.pdf

Pallarès, M., Vera, A. \& Tulla, A., (2018). Emprendimiento e innovación de las mujeres: hacia una mayor sostenibilidad en las áreas rurales de montaña. Cuadernos geográficos de la Universidad de Granada, 57 (3), 36-57. https:// revistaseug.ugr.es/index.php/cuadgeo/article/view/5770

Pérez, N., \& Madera, P. (2019). El emprendimiento en la población femenina afrodescendiente del corregimiento de La Boquilla. Aglala, 10(2), 81-98. https://revistas.curn.edu.co/index.php/aglala/article/view/1435

Pérez, A., \& Tallón, M. (2017). Estudio de la mujer emprendedora: El medio rural como oportunidad. RA \& DEM Revista de Administración y Dirección de empresas. https://dialnet.unirioja.es/servlet/articulo?codigo=6341525

Pérez, M., Vázquez, V. \& Zapata, E. (2008). Empoderamiento de las mujeres indígenas de Tabasco: El papel de los fondos regionales de la CDI. Cuicuilco, 15(42), 165-179. http://www.scielo.org.mx/scielo.php?script=sci_arttext\&pid=S0185-16592008000400010

Podsakoff, MacKenzie, Bachrach, \& Podsakoff, (2005). The influence of management journals in the 1980s and 1990s. Strategic Management Journal, 26(5), 473-488.

Queirolo, G. (2009). Victoria Ocampo [1890-1979]: Cruces entre feminismo, clase y elite intelectual. Clio \& asociados, (13), 135-159. http://sedici.unlp.edu.ar/handle/10915/31014

Rodríguez, C., \& Muñoz, J. (2015). Participación laboral de las mujeres rurales chilenas: Tendencias, perfiles y factores predictores. Cuadernos de Desarrollo Rural, 12(75), 77-98. https://revistas.javeriana.edu.co/index.php/desarrolloRural/article/view/10370

Saavedra, M., \& Camarena, M. (2015). Retos para el emprendimiento femenino en américa latina. Revista Criterio Libre, 13(22). https://doi.org/10.18041/1900-0642/criteriolibre.2015v13n22.129

Saldanha, P., \& Signorini, H. (2016). Práticas de empoderamento feminino na América Latina. Revista de estudios sociales, (56), 80-90. https://www.researchgate.net/publication/301307310_Praticas_de_empoderamento_feminino_na_America_Latina

Santamaria, A. (2015). Del fogón a la "Chagra": Mujeres, liderazgo y educación intercultural en la Amazonía colombiana y en la Sierra Nevada de Santa Marta. Revista Lusófona de Educação, 31(31). https://www.redalyc.org/ pdf/349/34944227010.pdf

Santander, P., Fernández, R., \& Yáñez, D. (2016). Motivaciones y condicionantes contextuales en el emprendimiento liderado por mujeres chilenas. Revista de Ciencias Sociales (Ve), 22(2), 63-77. https://www.redalyc.org/ pdf/280/28049145006.pdf

Santos, F., Barroso, M., \& Guzmán, C. (2013). La economía global y los emprendimientos sociales. Revista de economía mundial, (35), 177-196. https://www.redalyc.org/pdf/866/86629567010.pdf 
Sánchez, S., \& Jiménez, M. (2013). Mujeres rurales y participación social: análisis del asociacionismo femenino en la provincia de Granada (España). Cuadernos de Desarrollo Rural, 10(72), 223-242. http://www.scielo.org.co/pdf/ cudr/v10n72/v10n72a12.pdf

Vega, C., \& Bermúdez, H. (2019). Informalidad, emprendimiento y empoderamiento femenino. Economía popular y paradojas de la venta directa en el sur de Quito (Ecuador). Revista de Antropología Social, 28(2), 345. https:// revistas.ucm.es/index.php/RASO/article/view/65618

Viveros, M. (2017). La antropología colombiana el género y el feminismo. Revista Colombiana de Antropología -Maguaré, 31(2), 19-60. https://revistas.unal.edu.co/index.php/maguare/article/viewFile/71518/65677 